\title{
Ethical Implications of the Fecal Microbiota Transplantation: Disclosure of a False-Positive HIV Test
}

\author{
Caitlyn Hollingshead (D, Jacob Ciricillo, and Joel Kammeyer \\ The University of Toledo, 3000 Arlington AveToledo, $\mathrm{OH} 43614$, USA \\ Correspondence should be addressed to Caitlyn Hollingshead; caitlyn.hollingshead@utoledo.edu
}

Received 11 October 2020; Revised 31 July 2021; Accepted 24 November 2021; Published 6 December 2021

Academic Editor: Salim Surani

Copyright (c) 2021 Caitlyn Hollingshead et al. This is an open access article distributed under the Creative Commons Attribution License, which permits unrestricted use, distribution, and reproduction in any medium, provided the original work is properly cited.

Fecal microbiota transplantation (FMT) has gained popularity as an effective therapeutic option for Clostridioides difficile infection (CDI). Since its FDA recognition as a treatment modality for recurrent CDI in 2013, screening protocols for FMT donor stool have been in flux. However, extensive health questionnaires, in combination with serological and stool assays, have become mainstays in the donor screening process, although ethical implications are yet to be thoroughly considered. Herein, we present the case of a family member found to have a false-positive HIV test during the donor screening process and discuss potential ethical ramifications associated with FMT stool donation.

\section{Introduction}

Recurrent Clostridioides difficile infection (CDI) has established itself as a leading cause of infectious antibiotic-associated diarrhea worldwide, recurring in $20-30 \%$ of CDI cases [1]. Due to the increasing incidence of recurrent CDI and its evolving burdens on the healthcare system, fecal microbiota transplantation (FMT) has gained popularity as a therapeutic option for recurrent $\mathrm{CDI}[2,3]$. A recent meta-analysis exploring the efficacy of FMT, including thirty-seven studies, revealed clinical resolution of infection in $92 \%$ of cases of recurrent and refractory CDI [4]. Patients with multiple CDI episodes refractory to antibiotic therapy are candidates to undergo FMT, which has demonstrated decreased CDI-related mortality in hospitalized patients with recurrent CDI $[5,6]$. Choosing a family member as a donor can be advantageous due to similarities in microbiota and cost considerations. However, the screening process may present hereunto unconsidered ethical ramifications.

\section{Case Presentation}

A 54-year-old male was identified as an appropriate potential donor for fecal transplantation. His father, hospitalized for a fourth recurrence of $C$. difficile colitis after multiple failed antibiotic courses, was referred for FMT. As part of routine screening to evaluate his suitability as a donor, the son's HIV p24 antigen returned positive. The son was informed of his positive result by medical staff and was referred to infectious diseases without counseling. The son was in a monogamous relationship with his wife and had no risk factors for HIV infection. Confirmatory testing using a Multispot HIV-1 and HIV-2 antibody differentiation assay returned as negative, and subsequent HIV viral load was negative. The son was then counseled that he had a false-positive HIV test by an infectious diseases physician.

\section{Discussion}

FMT was first utilized as an accepted treatment modality for C. difficile colitis in the United States in 2013 when it gained FDA approval. However, it was first utilized for the treatment of pseudomembranous colitis in 1958 [7]. Its first use for a noninfectious cause was noted in 1989 when it was used for a patient with ulcerative colitis [7]. There are no universally accepted screening protocols for stool donors, but European guidelines recommend screening potential donors for HIV, cytomegalovirus, Epstein-Barr virus, hepatitis A, hepatitis B, hepatitis C, hepatitis E, syphilis, and Entamoeba histolytica [8]; see also Figure 1. 


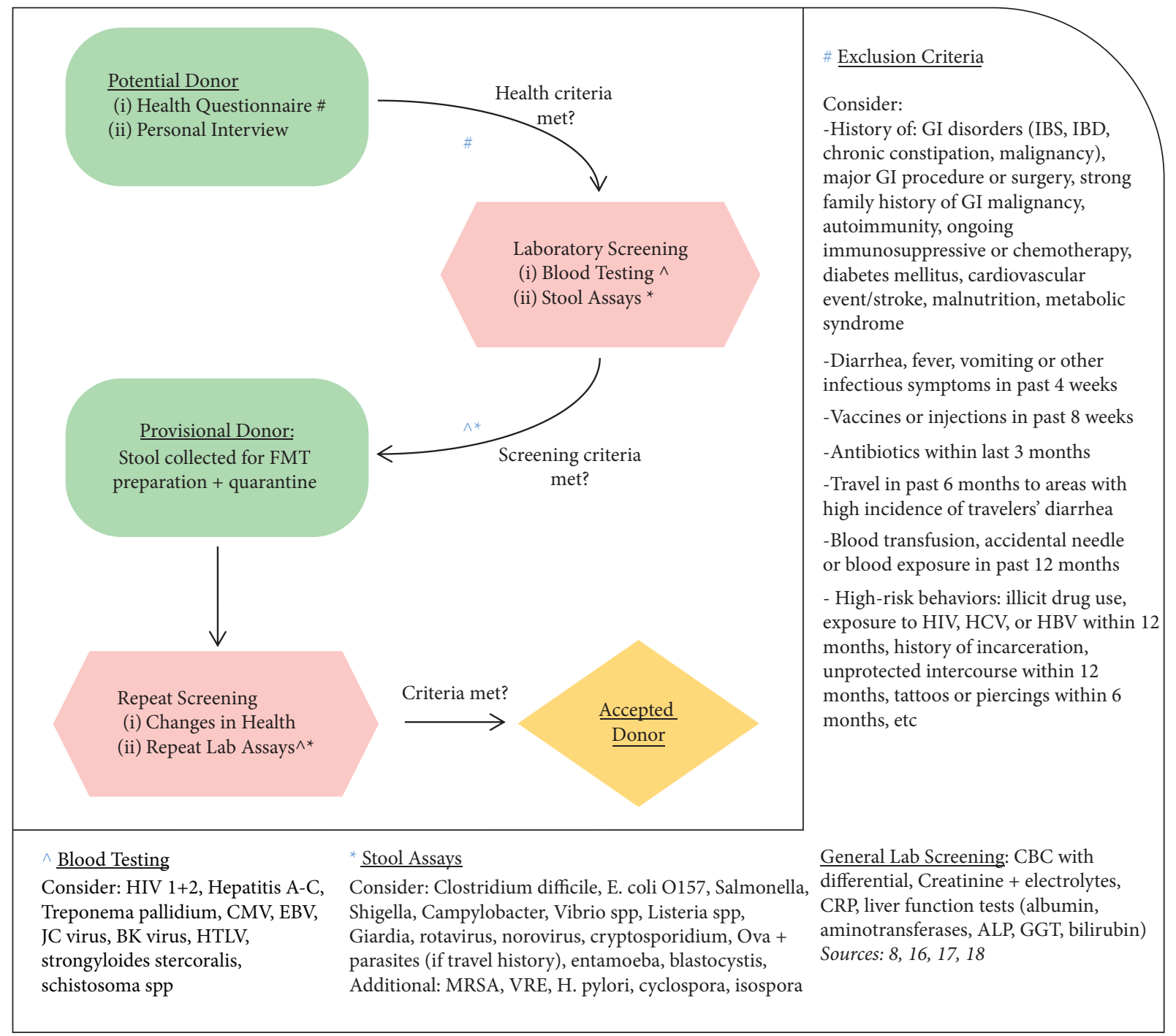

FiguRE 1: Summary of the FMT donor screening process: including the review of exclusion criteria, blood testing, and stool assays undergone by potential donors prior to authorization as an accepted donor. Note that these criteria are to be used as guidance for donor selection. However, this list of screening assays is not exhaustive, and additional testing may be considered in certain clinical scenarios.

As FMT is a relatively new treatment modality, new ethical considerations are raised regarding how potential donors with positive screening tests are best handled. In this case, the patient was informed he had HIV without receiving counseling, and further testing revealed this to be a falsepositive result. This disclosure imposes consequences, including potential isolation from the spouse or family and significant stigma. Further ethical considerations are raised upon consideration of what would have transpired had the result truly been positive: the physician must inform the father that his son was not a compatible match while protecting the son's autonomy.

Procuring stool from a stool bank from prescreened donors can cost between $\$ 1500$ and $\$ 2000$ [9]; therefore, choosing a family member as a donor is an attractive alternative due to decreased cost. Screening any potential donor for transmittable infectious diseases is an important step before donor selection. Several organizations have developed screening recommendations for donors that include HIV screening [10]. However, those ordering testing in this circumstance may not be well versed in counseling after a positive screening test. Current HIV testing has an extremely high specificity; however, in populations of low prevalence, the positive predictive value is extremely low [11]. Those involved in the screening of FMT donors should have procedures in place to provide counseling after positive results.

Many obstacles prevent FMT from being more frequently utilized for CDI [12]. Challenges include donor recruitment and consistent regulation of donor stool screening, with a recent example demonstrating only $3 \%$ of candidate donors ultimately qualifying for donation after screening [13]. The utilization of stool banks would have avoided the ethical dilemma in this case. They provide reliable donors and spare time spent undergoing prescreening while avoiding the risk of false-positive tests and the 
associated psychological harm to their family members. However, there have been recent reports of transmission of enteropathogenic E. coli and Shiga toxin-producing E. coli via FMT after donor stool was obtained from stool banks [14], which bring into question their screening practices.

Available data reveal excellent short-term safety data with FMT. However, it should be noted that most currently available randomized controlled studies have small sample sizes and short follow-up periods of eight to twelve weeks, making the long-term complications of FMT unknown [15]. In lieu of utilization of a stool bank, robust evidence-based guidelines for the screening of FMT donors are needed, as well as more randomized controlled studies with longer follow-up periods to demonstrate long-term safety data. Additionally, future guidelines regarding the usage of this modality should include guidance for gastroenterologists regarding disclosure and confirmation of any positive results of screening for infectious diseases of potential donor samples.

\section{Data Availability}

We have no underlying data to reference.

\section{Conflicts of Interest}

The authors declare that they have no conflicts of interest.

\section{References}

[1] C. H. Chilton, D. S. Pickering, and J. Freeman, "Microbiologic factors affecting Clostridium difficile recurrence," Clinical Microbiology and Infections, vol. 24, no. 5, pp. 476-482, 2018.

[2] G. K. Ma, C. M. Brensinger, Q. Wu, and J. D. Lewis, "Increasing incidence of multiply recurrent Clostridium difficile infection in the United States," Annals of Internal Medicine, vol. 167, no. 3, pp. 152-158, 2017.

[3] F. C. Lessa, Y. Mu, W. M. Bamberg et al., "Burden of Clostridium difficile infection in the United States," New England Journal of Medicine, vol. 372, no. 9, pp. 825-834, 2015.

[4] M. N. Quraishi, M. Widlak, N. Bhala et al., "Systematic review with meta-analysis: the efficacy of faecal microbiota transplantation for the treatment of recurrent and refractory Clostridium difficile infection," Alimentary Pharmacology and Therapeutics, vol. 46, no. 5, pp. 479-493, 2017.

[5] L. C. McDonald, D. N. Gerding, S. Johnson et al., "Clinical practice guidelines for Clostridium difficile infection in adults and children: 2017 update by the infectious diseases society of America (IDSA) and society for healthcare epidemiology of America (SHEA)," Clinical Infectious Diseases, vol. 66, no. 7, pp. 987-994, 2018.

[6] Y.-W. Cheng, X. Huiping, P. Emmalee et al., "Fecal microbiota transplant decreases mortality in patient with refractory severe and severe complicated Clostridium difficile infection not eligible for colectomy: 2017 fellows-in-training award (colon category)," American Journal of Gastroenterology, vol. 112, 2017.

[7] J.-W. Wang, C.-H. Kuo, F.-C. Kuo et al., "Fecal microbiota transplantation: review and update," Journal of the Formosan Medical Association, vol. 118, pp. S23-S31, 2019.
[8] G. Cammarota, G. Ianiro, H. Tilg et al., "European consensus conference on faecal microbiota transplantation in clinical practice," Gut, vol. 66, no. 4, pp. 569-580, 2017.

[9] "Treatment information. OpenBiome," 2019, https://www. openbiome.org/treatment-information. Accessed November 18, 2019.

[10] M. H. Woodworth, E. M. Neish, N. S. Miller et al., "Laboratory testing of donors and stool samples for fecal microbiota transplantation for recurrent Clostridium difficile infection," Journal of Clinical Microbiology, vol. 55, no. 4, pp. 1002-1010, 2017.

[11] S. Kim, J.-H. Lee, J. Y. Choi, J. M. Kim, and H.-S. Kim, "Falsepositive rate of a "fourth-generation" HIV antigen/antibody combination assay in an area of low HIV prevalence," Clinical and Vaccine Immunology, vol. 17, no. 10, pp. 1642-1644, 2010.

[12] J. R. Allegretti, B. H. Mullish, C. Kelly, and M. Fischer, "The evolution of the use of faecal microbiota transplantation and emerging therapeutic indications," The Lancet, vol. 394, no. 10196, pp. 420-431, 2017.

[13] Z. Kassam, N. Dubois, B. Ramakrishna et al., "Donor screening for fecal microbiota transplantation," New England Journal of Medicine, vol. 381, no. 21, pp. 2070-2072, 2019.

[14] "Fecal microbiota for transplantation: safety alert-risk of serious adverse events likely due to transmission of pathogenic organisms," Updated March 12, 2020. Accessed July 30, 2021. https://www.fda.gov/safety/medical-product-safetyinformation/fecal-microbiota-transplantation-safety-alertrisk-serious-adverse-events-likely-due-transmission, 2020.

[15] X. Tan and S. Johnson, "Fecal microbiota transplantation (FMT) for C. difficile infection, just say 'No'," Anaerobe, vol. $60,2019$.

[16] B. H. Mullish, M. N. Quraishi, J. P. Segal et al., "The use of faecal microbiota transplant as treatment for recurrent or refractory Clostridium difficile infection and other potential indications: joint British Society of Gastroenterology (BSG) and Healthcare Infection Society (HIS) guidelines," Gut, vol. 67, no. 11, pp. 1920-1941, 2018.

[17] M. H. Woodworth, C. Carpentieri, K. L. Sitchenko, and C. S. Kraft, "Challenges in fecal donor selection and screening for fecal microbiota transplantation: a review," Gut Microbes, vol. 8, no. 3, pp. 225-237, 2017.

[18] "The OpenBiome quality \& safety program. OpenBiome," 2021, https://staticl.squarespace.com/static/ 50e0c29ae4b0a05702af7e6a/t/605890d58d9fe90a81ad27fa/ $1616416981804 /$ The+OpenBiome+Quality $+\%$ 26+Safety+Program.pdfAccessed September 24, 2021. 